\title{
Depredación del lobo marino común Otaria flavescens sobre el pingüino de penacho amarillo Eudyptes $c$. chrysocome en isla Gonzalo, Diego Ramírez, sur de Chile
}

\author{
Predation of South American sea lion Otaria flavescens on Southern Rockhopper penguin \\ Eudyptes c. chrysocome in Gonzalo Island, Diego Ramírez, southernmost Chile
}

\author{
Jaime A. Cursach ${ }^{1,2,3}$, Cristián G. Suazo ${ }^{4}$ y Jaime R. $\operatorname{Rau}^{1,2,5}$
}

\begin{abstract}
${ }^{1}$ Programa de Doctorado en Ciencias mención Conservación y Manejo de Recursos Naturales, Universidad de Los Lagos, casilla 557, Puerto Montt, Chile. jcurval@gmail.com

${ }^{2}$ Laboratorio de Ecología, Departamento de Ciencias Biológicas y Biodiversidad, Universidad de Los Lagos, casilla 933, Osorno, Chile

${ }^{3}$ Laboratorio de Investigación Socioambiental, Programa ATLAS, Departamento de Ciencias Sociales, Universidad de Los Lagos, casilla 933, Osorno, Chile

${ }^{4}$ Albatross Task Force - Chile, BirdLife International

${ }^{5}$ Programa IBAM, Universidad de Los Lagos, casilla 933, Osorno, Chile
\end{abstract}

\begin{abstract}
During the summer season 2010-2011 was studied the predation of Otaria flavescens on Eudyptes chrysocome chrysocome in Gonzalo Island ( $56^{\circ} 31^{\prime} \mathrm{S} ; 68^{\circ} 42^{\prime} \mathrm{W}$ ), Diego Ramírez archipelago, southern Chile. In total, 75 attacks of sea lion on penguins were recorded, from which $34(45.3 \%)$ corresponded to successful attacks and kill of penguins. Among recorded behaviors during attacks, we highlight: i) persecutions during penguin's swimming, ii) persecutions at land and iii) surprise attacks under water. We identified a bird assemblage associated to predation events, dominated by Macronectes giganteus.
\end{abstract}

Key words: Attacks, behavior, sub-Antarctic Islands

\section{INTRODUCCIÓN}

El lobo marino común Otaria flavescens (Shaw, 1800) es la especie de pinnípedo más frecuente en el litoral chileno, con una distribución geográfica que abarca desde el norte de Perú hasta el Cabo de Hornos en el Pacífico y por el Atlántico hasta el sur de Brasil, incluyendo las islas Malvinas (Sielfeld 1999, Campagna 2008). Su distribución reproductiva occidental comprende por el norte desde isla Lobos de Tierra (6 $6^{\circ} 26^{\prime} S$ ) en Perú, hasta el archipiélago Diego Ramírez (56 31'S) en aguas subantárticas de Chile (Schlatter \& Riveros 1997, Campagna 2008).

La dieta de este mamífero marino es amplia y ha sido caracterizada por peces, cefalópodos, crustáceos, moluscos y desechos de la pesca (George-Nascimento et al. 1985, Thompson et al. 1998, Alonso et al. 2000, Suarez et al. 2005, Campagna 2008). También se ha registrado una conducta de alimentación oportunista sobre aves marinas, en especial sobre pingüinos (Raya-Rey et al. 2012). Entre las especies de pingüinos consumidos por el lobo marino común destaca el pingüino de penacho amarillo Eudyptes chrysocome chrysocome (Forster, 1781), ave marina que habita aguas costeras y pelágicas subantárticas, alimentándose principalmente de peces y krill Euphausia sp. (BirdLife International 2012, Raya-Rey et al. 2012). En Chile, la población reproductiva de este pingüino se estima en 378.321 parejas (que corresponde al 50\% de la población mundial de esta especie), de las cuales gran parte se concentra en el archipiélago Diego Ramírez, donde nidifica desde octubre a marzo de cada año (Kirkwood et al. 2007).

El objetivo de esta nota fue reportar por primera vez la depredación del lobo marino común sobre el pingüino de penacho amarillo, en el archipiélago Diego Ramírez, sur de Chile.

\section{Materiales Y MÉTODOS}

Desde el 29 de noviembre de 2010 hasta el 9 de enero de 2011, se realizaron observaciones de la depredación del lobo marino común (en adelante LMC) sobre el pingüino de penacho amarillo (en adelante PPA), en isla Gonzalo 
(56³1'21,2”S; 6842’56,4”O), archipiélago Diego Ramírez, sur de Chile.

Las observaciones fueron realizadas en una pequeña bahía ubicada en la costa noreste de isla Gonzalo, que los PPA utilizan para ingresar al mar desde sus sitios de nidificación (ubicados en las laderas del sector). En esta bahía se delimitó (a partir de sus características geográficas) un área aproximada de 0,18 ha, que abarcó una playa de cantos rodados, una zona intermareal rocosa y el submareal. Desde un punto fijo ubicado sobre un risco a 15 m.s.n.m., que cubrió visualmente toda el área señalada (coordenadas central: 56³1'16,85' S; $68^{\circ} 42^{\prime} 48,29$ 'O), se realizaron 13 sesiones de observación (Tabla 1) en las cuales se registró el número de $\mathrm{LMC}$, el número de PPA y el número de ataques de LMC sobre PPA. De igual forma, se tomó atención en las conductas de ataques desplegadas por el LMC, así como también en el ensamble de aves y mamíferos marinos presentes en el área de estudio.

Detalles de horarios y fechas de registro se presentan en Tabla 1. El esfuerzo de observación global fue de 865 $\min$.

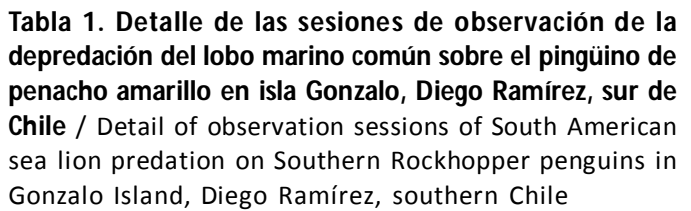

\begin{tabular}{ccc}
\hline $\begin{array}{c}\text { Número de } \\
\text { sesión }\end{array}$ & Fecha & Horario (h) \\
\hline 1 & $29 / 11 / 2010$ & $19: 20-20: 10$ \\
2 & $02 / 12 / 2010$ & $18: 00-19: 45$ \\
3 & $04 / 12 / 2010$ & $16: 30-18: 10$ \\
4 & $10 / 12 / 2010$ & $18: 00-19: 04$ \\
5 & $12 / 12 / 2010$ & $12: 20-13: 00$ \\
6 & $15 / 12 / 2010$ & $12: 00-13: 07$ \\
7 & $16 / 12 / 2010$ & $11: 10-12: 10$ \\
8 & $17 / 12 / 2010$ & $19: 02-20: 16$ \\
9 & $22 / 12 / 2010$ & $09: 40-11: 10$ \\
10 & $25 / 12 / 2010$ & $17: 15-18: 15$ \\
11 & $27 / 12 / 2010$ & $20: 00-20: 40$ \\
12 & $02 / 01 / 2011$ & $15: 50-17: 00$ \\
13 & $09 / 01 / 2011$ & $18: 15-19: 00$
\end{tabular}

\section{Resultados Y Discusión}

El área de estudio fue un sitio de importancia para el regreso de los PPA desde el mar hacia sus sitios de nidificación (Tabla 2), utilizando principalmente la playa de cantos rodados para salir del mar y comenzar a ascender entre las plantas de coirón Poa flabellata hacia las abruptas laderas de la isla, en donde se ubican sus nidos. Por otra parte, una fracción menor de PPA utilizó el área para ingresar al mar, debido a que con mayor frecuencia recurrieron a una puntilla rocosa ubicada al sur del área para entrar al mar.

El número de LMC observados dentro del área varió de 1 a 6 individuos, con una moda de 1 LMC por sesión de observación (Tabla 2). A través del transcurso de las sesiones de observación, se logró diferenciar mediante morfología, coloración y marcas en la piel, tal como han señalado Oliva et al. (2008) a 2 LMC adultos y un juvenil que frecuentaron el área para cazar PPA, identificando a uno de los individuos adultos como macho. En una sesión se registró la presencia de 2 LMC jóvenes, que desplegaron conductas de juego dentro del área, permaneciendo en el lugar para luego retirarse juntos. En 2 sesiones se observó que individuos solitarios merodearon el área, continuando su nado hacia la zona sur de la isla en donde se ubican las colonias de LMC.

En total, dentro del área se registraron 75 ataques de LMC sobre el PPA, de los cuales $34(45,3 \%)$ resultaron en ataques exitosos de depredación (Tabla 2).

Dentro de las conductas de ataque observadas en el LMC sobre el PPA, se registraron persecuciones en nado, persecuciones en tierra y ataques por sorpresa bajo el agua.

Las persecuciones en nado variaron en su tiempo de prolongación, destacando un seguimiento de caza que duró $20 \mathrm{~s}$, donde un individuo de PPA logró escapar del depredador mediante rápidos y zigzagueantes movimientos hasta alcanzar la playa. Los ataques exitosos se caracterizaron por la captura de PPA mediante el hocico del depredador, consumiendo la zona abdominal de la presa y dejando los restos en el agua, los cuales fueron posteriormente consumidos por aves marinas en la superficie del mar. En una sesión hubo 2 LMC dentro del área, observándose que tras uno de ellos realizar un ataque exitoso, su presa fue arrebatada por el otro LMC (mediante nado) que luego de quitar y consumir la presa se dirigió hacia la zona sur del área en donde inmediatamente realizó un ataque exitoso propio. Mientras que el LMC al cual se le arrebató su presa, se mantuvo 
Tabla 2. Resultados de las sesiones de observación de la depredación del lobo marino común sobre el pingüino de penacho amarillo (PPA) en isla Gonzalo, Diego Ramírez, sur de Chile / Results of observation sessions of South American sea lion predation on Southern Rockhopper penguins in Gonzalo Island, Diego Ramírez, southern Chile

\begin{tabular}{|c|c|c|c|c|c|c|}
\hline $\begin{array}{l}\text { Número } \\
\text { de sesión }\end{array}$ & $\begin{array}{l}\text { Tiempo de } \\
\text { observación } \\
\text { (min) }\end{array}$ & $\begin{array}{l}\mathrm{N}^{\mathrm{o}} \text { lobos } \\
\text { marinos }\end{array}$ & $\begin{array}{l}\mathrm{N}^{\circ} \text { de PPA que } \\
\text { ingresan al mar }\end{array}$ & $\begin{array}{l}\mathrm{N}^{\circ} \text { de PPA que } \\
\text { regresan del mar }\end{array}$ & $\begin{array}{l}\mathrm{N}^{\circ} \text { total de } \\
\text { ataques }\end{array}$ & $\begin{array}{l}\mathrm{N}^{0} \text { de } \\
\text { ataques } \\
\text { exitosos }\end{array}$ \\
\hline 1 & 50 & 6 & 12 & 131 & 27 & 15 \\
\hline 2 & 105 & 2 & 0 & 127 & 4 & 1 \\
\hline 3 & 100 & 1 & 20 & 104 & 3 & 1 \\
\hline 4 & 64 & 1 & 0 & 90 & 0 & 0 \\
\hline 5 & 40 & 2 & 0 & 51 & 6 & 3 \\
\hline 6 & 67 & 2 & 0 & 98 & 11 & 6 \\
\hline 7 & 60 & 1 & 0 & 72 & 0 & 0 \\
\hline 8 & 74 & 3 & 16 & 148 & 9 & 5 \\
\hline 9 & 90 & 1 & 22 & 277 & 11 & 1 \\
\hline 10 & 60 & 1 & 2 & 174 & 1 & 0 \\
\hline 11 & 40 & 0 & 13 & 101 & 0 & 0 \\
\hline 12 & 70 & 1 & 56 & 418 & 0 & 0 \\
\hline 13 & 45 & 1 & 45 & 205 & 3 & 2 \\
\hline
\end{tabular}

segregado en el margen norte del área donde posteriormente realizó un ataque exitoso.

Las persecuciones en tierra consistieron en la continuación de seguimientos en nado donde el LMC persistió acechando al PPA, hasta lograr capturarlo en la playa. Estas capturas fueron realizadas en tierra, donde el LMC atrapó PPA mediante su hocico, para posteriormente regresar al mar y concretar el consumo de la presa capturada. Un segundo tipo de ataque en tierra, consistió en que el LMC directamente salió a la superficie por entre las rocas de la zona intermareal (porción sur del área), para atacar a los PPA presentes en la franja supramareal (rocosa), saliendo del área de observación limitando el registro de ataques exitosos. En una de las sesiones se observó a un LMC fracasar en su intento por subir a la zona intermareal, debido a que en dicho momento existió marea baja en la zona, limitando el acceso al depredador.

Los ataques por sorpresa bajo el agua consistieron en apariciones del LMC por debajo del grupo de PPA que ingresaban en nado al área, capturando la presa mediante mordida y posterior movimiento de cabeza (en forma de abanico), desgarrando en el aire el cuerpo del PPA, sumergiéndose con la presa y emergiendo masticando su alimento. Un segundo tipo de ataque por sorpresa fue la conducta de mantener puntos fijos de espera bajo el agua, posicionándose tanto en aguas abiertas como entre las abundantes frondas del alga Durvillaea antarctica, (Chamisso) Hariot, realizando desde estos puntos ataques sorpresivos a los PPA que transitaron en la cercanía. Se registró un tiempo de espera (inmersión) promedio de 34 \pm 20 s (rangos de 21 a 80; $\mathrm{n}=7$ ).

Tras cada ataque exitoso, los restos de PPA no consumidos por el LMC (i.e., cabeza, vértebras, patas y aletas) fueron alimento para aves marinas que constituyeron un ensamble dominado por el petrel gigante antártico Macronectes giganteus (Gmelin, 1785), seguido por el petrel damero Daption capense (Linnaeus, 1758) y la gaviota dominicana Larus dominicanus (Lichtenstein, 1823). Se registró en menor frecuencia al petrel gigante subantártico Macronectes halli (Mathews, 1912) y el yeco 
Phalacrocorax brasilianus (Gmelin, 1789). A excepción de $D$. capense y $M$. halli, todas estas aves nidifican en el archipiélago Diego Ramírez (Schlatter \& Riveros 1997).

Existieron sesiones de observación en las cuales se registró la presencia de un individuo macho juvenil de elefante marino Mirounga leonina (Linnaeus, 1758) descansando en la playa del área de estudio, así como también de una hembra de la misma especie que transitó mediante nado por el margen del área de estudio. En ambos casos no se registraron ataques sobre el PPA. En otra sesión, se observó a un individuo macho adulto del lobo fino austral Arctocephalus australis (Zimmerman, 1783) ascender a la zona supramareal del área (porción sur) sin realizar ataques sobre el PPA, pero presentando una conducta inquieta de intención por perseguir a los PPA, los cuales al instante ya habían alcanzado zonas altas de seguridad imposibles de llegar para el lobo fino austral, el cual descansó sobre las rocas. El lobo fino austral se reproduce localmente en isla Gonzalo y el archipiélago Diego Ramírez, mientras que el elefante marino es considerado como un visitante común en islas Diego Ramírez (Schlatter \& Riveros 1997).

Las conductas de ataque del LMC observadas en isla Gonzalo, concuerdan con las realizadas por Raya-Rey et al. (2012) en bahía Franklin, isla de Los Estados, Tierra del Fuego. De forma similar, el LMC utilizó las frondas de D. antarctica como una estructura para ocultarse en la columna de agua y atacar por sorpresa a los PPA que transitaron por su cercanía. Por otra parte, las observaciones de ataques del LMC sobre el PPA mediante persecuciones en tierra, se caracterizaron en que el LMC no consumió su presa sino hasta regresar al mar, devorando principalmente la masa abdominal del PPA.

La depredación del LMC sobre el PPA, genera residuos o restos de la presa que no son consumidos por el depredador, los cuales permanecen flotando en el agua, ocasión que es aprovechada por las aves marinas que las consumen como carroña. Tal como lo registrado por RayaRey et al. (2012), el ensamble de aves asociadas a la depredación del LMC sobre el PPA fue dominado por el petrel gigante antártico.

Una temática de investigación que permitiría desarrollar el conocimiento existente hasta ahora sobre la interacción depredador-presa descrita en el presente trabajo, sería evaluar: i) la existencia de individuos exclusivos (e.g., pre-adultos de LMC) que depredan sobre el PPA, ii) la coexistencia de otros sitios donde ocurran estos ataques en isla Gonzalo y el resto del archipiélago Diego Ramírez, iii) la existencia de individuos exclusivos (e.g., por edad o estado reproductivo del PPA) que son depredados por el LMC, iv) competencia intraespecífica del LMC por depredar al PPA, v) competencia interespecífica entre mamíferos marinos por depredar al PPA.

Finalmente, es importante destacar que la interacción depredador-presa existente entre mamíferos y aves marinas permite esquematizar el traspaso energético en tramas tróficas del ecosistema marino (Estes et al. 1998), i.e., la productividad del mar Antártico sostiene la abundancia de krill, componente clave del ecosistema, sustentando gran parte de la biodiversidad animal (Marín $\&$ Delgado 1998), el cual es consumido por el PPA que luego de alimentarse retorna en nado hacia la isla subantártica en donde nidifica, situación que es aprovechada por el LMC, depredando al PPA en su intento por llegar a tierra y generando carroña que es utilizada por petreles y gaviotas.

\section{Agradecimientos}

A la Universidad de Los Lagos por financiar los costos de esta publicación. A la Armada de Chile por el apoyo logístico. CG Suazo agradece el apoyo del Pacific Seabird Group. A dos revisores anónimos de la RBMO.

\section{LITERATURA CITADA}

Alonso KM, EA Crespo, SN Pedraza, NA García \& MA Coscarella. 2000. Food habits of the South American sea Lion, Otaria flavescens, of Patagonia, Argentina. Fishery Bulletin 98: 250-263.

BirdLife International. 2012. Eudyptes chrysocome. IUCN Red list of threatened species. <www.iucnredlist.org>

Campagna C. 2008. Otaria flavescens. IUCN Red list of threatened species. <www.iucnredlist.org>

Estes JA, MT Tinker, TM Williams \& DF Doak. 1998. Killer whale predation on sea otters linking oceanic and nearshore ecosystems. Science 282: 473-476.

George-Nascimento M, R Bustamante \& C Oyarzún. 1985. Feeding ecology of the South American sea lion Otaria flavescens: food contents and food selectivity. Marine Ecology Progress Series 21: 135-143.

Kirkwood R, K Lawton, C Moreno, J Valencia, R Schlatter \& G Robertson. 2007. Estimates of Southern Rockhopper and Macaroni Penguin numbers at the Ildefonso and Diego Ramirez Archipelagos, Chile, using quadrat and distancesampling techniques. Waterbirds 30: 259-267.

Marín VH \& LE Delgado. 1998. La Antártica, 90 pp. Editorial Universitaria, Santiago. 
Oliva D, W Sielfeld, M Buscaglia, M Matamala, R Moraga, H Pavés, MJ Pérez, D Schrader, M Sepúlveda \& A Urra. 2008. Plan de acción para disminuir y mitigar los efectos de las interacciones del lobo marino común (Otaria flavescens) con las actividades de pesca y acuicultura de la X y XI región. Informe Final, Proyecto FIP 2006-34: 1-256 pp. Subsecretaría de Pesca, Valparaíso. <http://www.fip.cl/FIP/ Archivos/pdf/informes/inffinal\%202006-34.pdf>

Raya-Rey A, R Sáenz-Samaniego \& PF Petracci. 2012. New records of South American sea lion Otaria flavescens predation on southern rockhopper penguins Eudyptes chrysocome at Staten Island, Argentina. Polar Biology 35: 319-322.

Schlatter RP \& G Riveros. 1997. Historia natural del Archipiélago Diego Ramírez, Chile. Serie Científica INACH 47: 87-112.
Sielfeld W. 1999. Estado del conocimiento sobre conservación y preservación de Otaria flavescens (Shaw, 1800) y Arctocephalus australis (Zimmermann, 1783) en las costas de Chile. Estudios Oceanológicos 18: 81-96.

Suarez AA, D Sanfelice, MH Cassini \& HL Cappozzo. 2005. Composition and seasonal variation in the diet of the South American sea lion (Otaria flavescens) from Quequén, Argentina. Latin American Journal of Aquatic Mammals 4: 163-174.

Thompson D, CD Duck, BJ McConnell \& J Garrett. 1998. Foraging behaviour and diet of lactating female southern sea lions (Otaria flavescens) in the Falklands Islands. Journal of Zoology 246: 135-146.

Recibido el 23 de septiembre de 2013 y aceptado el 14 de mayo de 2014

Editor: Claudia Bustos D. 